\title{
Rate-Control Drugs Affect Variability and Irregularity Measures of RR Intervals in Patients with Permanent Atrial Fibrillation
}

\author{
VALENTINA D.A. CORINO, PH.D., ${ }^{*}$ SARA R. ULIMOEN, M.D., $†$ STEVE ENGER, R.N., $\dagger$ \\ LUCA T. MAINARDI, Ph.D., ${ }^{*}$ ARNLJOT TVEIT, M.D., Ph.D., $\dagger$ and PYOTR G. PLATONOV, M.D., \\ PH.D., F.H.R.S. $\ddagger$
}

From the *Dipartimento di Elettronica, Informazione e Bioingegneria, Politecnico di Milano, Milano, Italy; $\dagger$ Department of Medical Research, Bærum Hospital, Vestre Viken Hospital Trust, Norway; and $\ddagger$ Department of Cardiology, Clinical Sciences, Center for Integrative Electrocardiology, Lund University, Lund, Sweden

\begin{abstract}
Heart Rate Variability and Irregularity During AF. Introduction: Irregularity measures have been suggested as risk indicators in patients with atrial fibrillation (AF); however, it is not known to what extent they are affected by commonly used rate-control drugs. We aimed at evaluating the effect of metoprolol, carvedilol, diltiazem, and verapamil on the variability and irregularity of the ventricular response in patients with permanent $\mathrm{AF}$.

Methods and Results: Sixty patients with permanent AF were part of an investigator-blind cross-over study, comparing 4 rate-control drugs (diltiazem, verapamil, metoprolol, and carvedilol). We analyzed five 20-minute segments per patient: baseline and the 4 drug regimens. On every segment, heart rate (HR) variability and irregularity of $R R$ series were computed. The variability was assessed as standard deviation, pNN20, pNN50, pNN80, and rMSSD. The irregularity was assessed by regularity index, approximate (ApEn), and sample entropy. A significantly lower HR was obtained with all drugs, the HR was lowest using the calcium channel blockers. All drugs increased the variability of ventricular response in respect to baseline (as an example, rMSSD: baseline $171 \pm 47$ milliseconds, carvedilol $229 \pm 58$ milliseconds; $P<0.05$ vs. baseline, metoprolol $226 \pm 66$ milliseconds; $P<0.05$ vs. baseline, verapamil $228 \pm 84 ; P$ $<0.05$ vs. baseline, diltiazem $256 \pm 87$ milliseconds; $P<0.05$ vs. baseline and all other drugs). Only $\beta$-blockers significantly increased the irregularity of the RR series (as an example, ApEn: baseline $1.86 \pm$ 0.13 , carvedilol $1.92 \pm 0.09 ; P<0.05$ vs. baseline, metoprolol $1.93 \pm 0.08 ; P<0.05$ vs. baseline, verapamil $1.86 \pm 0.22 \mathrm{~ns}$, diltiazem $1.88 \pm 0.16 \mathrm{~ns})$.

Conclusion: Modification of $\mathrm{AV}$ node conduction by rate-control drugs increase RR variability, while only $\beta$-blockers affect irregularity.
\end{abstract}

\section{Introduction}

Irregularity measures have been suggested as risk indicators in patients with atrial fibrillation (AF). The few studies analyzing variability and irregularity of the RR series showed that a reduced irregularity of $\mathrm{RR}$ intervals in permanent $\mathrm{AF}$ was associated with poor outcome..$^{1-4}$ The very first study by Yamada ${ }^{1}$ showed that a reduced RR irregularity in a 24-hour ambulatory ECG had an independent prognostic value for cardiac mortality during long-term follow-up in patients with

The RATAF study was supported by the South-Eastern Norway Regional Health Authority and by the Medical Research Foundation, Bærum Hospital, Norway. Dr. Corino received travel grants from the Swedish Heart-Lung Foundation. Dr. Platonov was supported by the Swedish Heart-Lung Foundation, donation funds at Skåne University Hospital, Lund (Sweden), and research funds form the Swedish National Healthcare System (ALF). Other authors: No disclosures.

Address for correspondence: Valentina D.A. Corino, Ph.D., Dipartimento di Elettronica, Informazione e Bioingegneria, Politecnico di Milano, Via Golgi 39, 20133, Milano, Italy. Fax: +39 22399 3360; E-mail: valentina.corino@polimi.it chronic AF. More recently, in a post hoc analysis, reduced variability of RR intervals during AF, likely caused by autonomic dysfunction, was found to be an independent predictor of all cause mortality in patients with left ventricular dysfunction following myocardial infarction. ${ }^{2}$ Reduced irregularity was an independent predictor of all cause mortality, as well as sudden death and heart failure progression in patients with mild to moderate heart failure. ${ }^{3}$ Despite the accumulating data that suggest potential use of irregularity measures as risk indicators in patients with $\mathrm{AF}$, it is not known to what extent it can be affected by commonly used rate-control drugs. In one earlier study, ${ }^{5}$ we did not observe significant differences in ventricular response irregularity measures in regard to the use of rhythm- or rate-control drugs in patients with $\mathrm{AF}$; however, this association has not been evaluated in controlled settings.

Recently, the RATe control in Atrial Fibrillation (RATAF) study compared the effects of 4 once-daily drug regimens on heart rate and arrhythmia related symptoms, in patients with permanent AF. ${ }^{6}$ The RATAF study was designed to compare 4 drug regimens (metoprolol, diltiazem, verapamil, and carvedilol) used to reduce the ventricular heart rate in patients with permanent AF.

The aim of this study was to evaluate the effect of metoprolol, carvedilol, diltiazem, and verapamil on the variability 
TABLE 1

Demographic Characteristics and Cardiovascular History in the Study Population

\begin{tabular}{lc}
\hline Variable & Value \\
\hline Age (years) & $71 \pm 9$ \\
Gender (male/female) & $42 / 18$ \\
AF duration (months) & $11(2-121)$ \\
Body mass index (kg/m ${ }^{2}$ ) & $27 \pm 4$ \\
Stroke or transitory ischemic attack & $7(12 \%)$ \\
Diabetes mellitus & $3(5 \%)$ \\
Hypertension & $25(42 \%)$ \\
Chronic obstructive pulmonary disease & $3(5 \%)$ \\
Systolic blood pressure (mmHg) & $141 \pm 18$ \\
Diastolic blood pressure (mmHg) & $91 \pm 10$ \\
Left atrial diameter (long-axis view, mm) & $50.4 \pm 6.6$ \\
Left ventricular ejection fraction (\%) & $61.4 \pm 7.5$ \\
Warfarin & $56(93 \%)$ \\
Aspirin & $4(7 \%)$ \\
Angiotensin receptor blocker or angiotensin-converting & $22(37 \%)$ \\
$\quad$ enzyme inhibitor & \\
Diuretics & $9(15 \%)$ \\
Statins & $12(20 \%)$ \\
\hline Values are expressed as mean &
\end{tabular}

Values are expressed as mean $\pm \mathrm{SD}$, median (range) or $\mathrm{n}(\%)$.

and irregularity of the ventricular rate in patients with permanent AF.

\section{Methods}

\section{Protocol}

The RATAF study was a prospective, randomized, investigator-blind, crossover study designed to compare 4 drug regimens used to reduce the ventricular heart rate in patients with permanent AF. Most patients were recruited from the AF outpatient clinic at Baerum Hospital (Baerum, Norway) from May 2006 to June 2010. Detailed protocol of the study is described elsewhere. ${ }^{6}$ The study protocol was approved by the regional ethics committee and the Norwegian medicines agency, and all patients signed informed consent. Clinical characteristics are shown in Table 1.

The participants received the following drug regimens in a randomized cross-over design: (i) metoprolol slowrelease tablets $100 \mathrm{mg} / \mathrm{day}$, (ii) diltiazem sustained-release capsules $360 \mathrm{mg} /$ day, (iii) verapamil modified-release tablets $240 \mathrm{mg} /$ day, and (iv) carvedilol immediate-release tablets 25 $\mathrm{mg} /$ day. Each drug was given for at least 3 weeks to ensure an adequate period of wash out of the previous treatment and steady-state plasma concentrations. Before starting the first treatment and at the last day of each of the 4 treatment periods, 24-hour Holter recordings were made. The patients were encouraged to maintain normal daily activity during the Holter registration.

\section{Ventricular Response Analysis}

We analyzed five 20-minute segments per patient: baseline and the 4 drug regimens, all starting at 2 p.m. (that was found to be the peak of the rate-reducing effect in Ref. (6)). Variability and irregularity measures automatically were computed; for a visual explanation of the difference between variability and irregularity of RR series see Figure 1 . When all the patterns in the time series are the same (as in the rows of Fig. 1), the irregularity is the same. The variability of the series depends on the absolute values; thus, variability can be same in series that have different irregularity.

\section{$R R$ variability}

Time domain analysis includes the heart rate (HR), the standard deviation (SD) of all normal RR intervals, the root of the mean squared differences of successive RR intervals (rMSSD) and the percentage of interval differences of successive RR intervals greater than 20 milliseconds (pNN20), 50 milliseconds (pNN50), and 80 milliseconds (pNN80). ${ }^{7}$

\section{$R R$ irregularity}

Irregularity of RR intervals was assessed by 3 nonlinear measures: approximate (ApEn) and sample (SampEn) entropy, and the regularity index $(\mathrm{R})$.

\section{Approximate and sample entropy}

The approximate entropy (ApEn) is a regularity statistic quantifying the unpredictability of fluctuations in a time series such as an instantaneous heart rate time series. The presence of repetitive patterns of fluctuation in a time series makes it more predictable than a time series in which such patterns are absent. ApEn reflects the likelihood that similar patterns of observations will not be followed by additional similar observations. A time series containing many repetitive patterns, that is, a regular and predictable series, has a relatively small ApEn; a less predictable, that is, more complex, process has a higher ApEn. ${ }^{8}$ The ApEn algorithm counts each sequence as matching itself, and this makes the ApEn biased. Therefore, the sample entropy (SampEn), not counting self-matches, has been introduced. ${ }^{9}$

\section{Regularity}

Conditional entropy may be used to estimate a regularity index, $\mathrm{R}$, defined as the degree of recurrence of a pattern in a signal. The conditional entropy represents the amount of information carried by the most recent sample of a normalized realization of the series when its past L-1 samples are known. The R tends to zero if the series is an unpredictable process and tends to one if the series is a periodic signal and it assumes intermediate values for those processes that can be partially predicted by the knowledge of the past samples. ${ }^{10}$

\section{Statistical Analysis}

All the computed parameters were estimated for every 20-minute segment. One-way repeated measures ANOVA test was performed to compare the computed parameters during baseline and drug regimens; if the $\mathrm{P}$ value of the ANOVA test was significant, a paired $t$-test or Wilcoxon test with Holm's correction was applied.

A $\mathrm{P}<0.05$ was considered statistically significant. All analyses and statistical tests were performed using MATLAB ${ }^{\circledR}$ R2012b (The MathWorks, USA).

\section{Results}

\section{Patient Characteristics and Data Availability}

Of the 80 selected patients, only the 60 patients who completed the 4 drug treatments were included in the study. Clinical characteristics are presented in Table 1. 


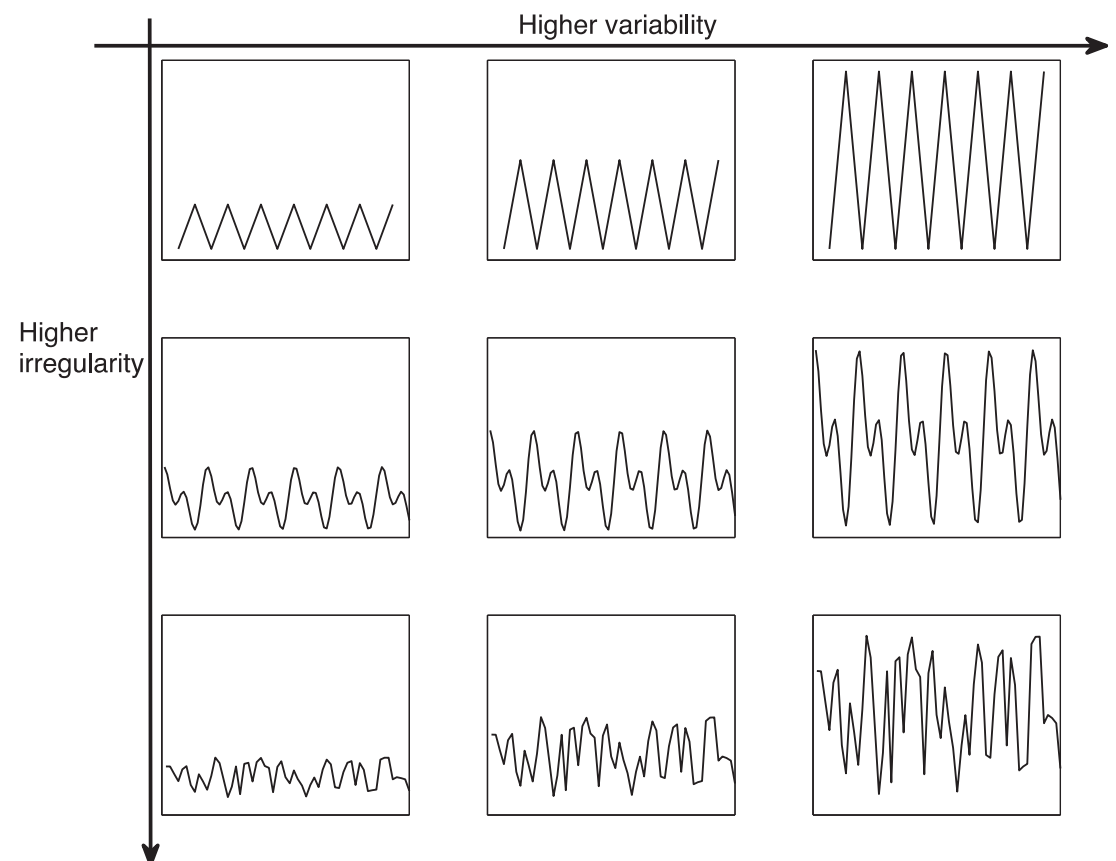

Figure 1. Figure explaining the difference between variability and irregularity in time series. Each row shows series with the same irregularity but increasing variability going from the left to the right, whereas each column shows series with the same variability but different increasing irregularity moving from the top to the bottom.

TABLE 2

Mean \pm SD for All Parameters During Baseline and Drug Administration

\begin{tabular}{|c|c|c|c|c|c|}
\hline & Baseline & Carvedilol & Metoprolol & Diltiazem & Verapamil \\
\hline HR (bpm) & $110 \pm 18$ & $88 \pm 14^{*}$ & $89 \pm 16^{*}$ & $79 \pm 16^{*, \dagger, \ddagger}$ & $78 \pm 18^{*, \dagger, \sharp}$ \\
\hline SD (milliseconds) & $133 \pm 37$ & $169 \pm 41^{*}$ & $166 \pm 48^{*}$ & $190 \pm 59^{*, \dagger, \ddagger}$ & $174 \pm 58^{*}$ \\
\hline pNN20 (\%) & $87 \pm 4$ & $91 \pm 3^{*}$ & $91 \pm 3^{*}$ & $90 \pm 9^{*}$ & $88 \pm 12$ \\
\hline pNN50 (\%) & $68 \pm 8$ & $77 \pm 6^{*}$ & $77 \pm 8^{*}$ & $77 \pm 13^{*}$ & $73 \pm 16$ \\
\hline pNN80 (\%) & $54 \pm 10$ & $65 \pm 8^{*}$ & $65 \pm 10^{*}$ & $66 \pm 14^{*}$ & $62 \pm 16^{*}$ \\
\hline rMSSD (milliseconds) & $171 \pm 47$ & $229 \pm 58^{*}$ & $226 \pm 66^{*}$ & $256 \pm 87^{*, \dagger, \ddagger}$ & $228 \pm 84^{*}, \S$ \\
\hline ApEn (a.u.) & $1.86 \pm 0.13$ & $1.92 \pm 0.09^{*}$ & $1.93 \pm 0.08^{*}$ & $1.88 \pm 0.16$ & $1.86 \pm 0.22$ \\
\hline SampEn (a.u.) & $1.68 \pm 0.18$ & $1.77 \pm 0.14^{*}$ & $1.79 \pm 0.12^{*}$ & $1.73 \pm 0.21$ & $1.70 \pm 0.30$ \\
\hline $\mathrm{R}$ (a.u.) & $0.10 \pm 0.06$ & $0.06 \pm 0.04^{*}$ & $0.07 \pm 0.04^{*}$ & $0.08 \pm 0.07$ & $0.10 \pm 0.08^{\dagger}$ \\
\hline
\end{tabular}

$* \mathrm{P}<0.05$ comparison with baseline.

${ }^{\dagger} \mathrm{P}<0.05$ comparison with carvedilol.

${ }^{\ddagger} \mathrm{P}<0.05$ comparison with metoprolol.

${ }^{\S} \mathrm{P}<0.05$ comparison with diltiazem.

\section{Rate-Control Drugs Effect on Variability and Irregularity}

Table 2 shows the results for all computed parameters. It can be observed that a significant reduction in HR is obtained with all drugs; moreover, the calcium channel blockers (diltiazem and verapamil) reduced the HR more than the $\beta$ blockers carvedilol and metoprolol. As shown in Figure 2A, during the 4 drug regimens there was a decrease in HR of about $20 \%$ for $\beta$-blockers (carvedilol $19 \pm 12 \%$, metoprolol $18 \pm 14 \%$ ) and $30 \%$ for calcium channel blockers (diltiazem $27 \pm 15 \%$, verapamil $28 \pm 18 \%$ ), as observed in Ref. (6).

From Table 2, it can be observed that all drugs increased the variability of ventricular response compared to baseline. Moreover, diltiazem was the drug inducing the highest increase in variability, resulting in a value of rMSSD significantly higher than with all the other drugs. On the contrary, it can be noted that only $\beta$-blockers increased the irregularity of the RR series, making the series significantly more irregular than at baseline, whereas the calcium channel blockers did not affect it. Figures $2 \mathrm{~B}$ and $\mathrm{C}$ show the percentage increase in rMSSD and SampEn, as an example of variability and irregularity measure, respectively. The increase in rMSSD is more evident for diltiazem, and the irregularity is increased mostly using $\beta$-blockers.

\section{Discussion}

To the best of our knowledge, this is the first time the effect of rate-control drugs on heart rate variability and irregularity is assessed in the setting of randomized prospective crossover designed study. We report significant differences between the 2 commonly used classes of rate-control drugs in regard to their effect on variability and irregularity of ventricular response in patients with permanent AF. Calcium channel blockers diltiazem and verapamil reduce HR, and increase time-domain measures of heart rate variability without effect on irregularity parameters. $\beta$-blockers carvedilol and metoprolol do not only reduce HR and increase timedomain measures of heart rate variability but also increase the irregularity parameters.

Long-term clinical benefit of modulation of variability and regularity of atrioventricular (AV) conduction during 



Figure 2. Boxplots of percentage of increase or decrease compared to baseline for (A) heart rate, (B) rMSSD (a variability measure), and (C) SampEn (an irregularity measure) during the 4 drug regimens.

$\mathrm{AF}$, apart from the effect of ventricular rate reduction, has not previously been demonstrated. Therefore, our findings should be interpreted as an attempt to clarify, in a controlled manner, the effect rate-control drugs have on AV conduction characteristics in order to assess reliability of RR variability and irregularity indices that appear to be linked to prognosis in patients with $\mathrm{AF}^{1-3}$ Reduced irregularity of the RR intervals in a 24-hour ambulatory ECG appeared to be an independent predictor of cardiac mortality during long-term follow-up in patients with chronic AF and mildly symptomatic congestive heart failure. ${ }^{1}$ A reduction in all ventricular response variability and irregularity measures was associated with an increased risk for cardiac death. After adjusting for clinical covariates, irregularity, but not the variability, measures had a predictive value for cardiac death. ${ }^{1}$

More recently, we analyzed variability and irregularity of RR intervals in patients with chronic AF with mild to moderate heart failure. During long-term follow-up, a reduced irregularity was observed in non-survivors. In particular, reduced ApEn was found to be a significant predictor of total mortality, sudden death and heart failure death in the univariate analysis as well as after adjustment for significant clinical covariates in a multivariate model. On the contrary, no differences were found in variability measures. ${ }^{3}$ In another recent study, we analyzed a subgroup of patients enrolled in the MADIT-II study with AF at baseline. A variability measure (pNN20) appeared to be an independent predictor of mortality in multivariate Cox analysis, whereas ApEn was not predictive of clinical outcome. However, there were important differences in the clinical profile of the ischemic patients with congestive heart failure enrolled in the MADIT-II study and the patients with permanent AF with more preserved left ventricular ejection fraction of the other studies. ${ }^{1,3}$

Interpretation of the prognostic impact of RR-irregularity measures is, however, rather complex since the majority of patients with permanent $\mathrm{AF}$ take rate-control medications. In our earlier study, ${ }^{5}$ we did not observe any difference in RRirregularity parameters during AF between patients with congestive heart failure in regard to the use of either rate-control, rhythm-control, or no antiarrhythmic drugs at baseline. The current study, in which rate-control drugs were administered in a controlled manner, demonstrates that RR-irregularity measures, which were significantly associated with the longterm outcome in earlier studies, seem to be unaffected by rate control using calcium channel blockers, whereas $\beta$-blockers significantly, even though rather modestly, increased them. This is in contrast with a previous study ${ }^{11}$ where we found no difference in irregularity after esmolol infusion. This disagreement may be due to the differences in the population size, route of administration and the type of $\beta$-blocker used in the 2 studies.

\section{Conclusion}

In this study, we analyzed the effect of the 4 common drugs for rate-control in patients with AF and we found that calcium channel blockers and $\beta$-blockers influenced $\mathrm{AV}$ node conduction differently. Both calcium channel blockers and $\beta$ blockers reduced HR and increased time-domain measures of heart rate variability, but only $\beta$-blockers increased the irregularity measures. Therefore, use of $\beta$-blockers should be adjusted for when assessing irregularity in AF patients, which has been suggested as a risk indicator in patients with AF.

\section{References}

1. Yamada A, Hayano J, Sakata S, Okada A, Mukai S, Ohte N, Kimura G: Reduced ventricular response irregularity is associated with increased mortality in patients with chronic atrial fibrillation. Circulation 2000;102:300-306.

2. Platonov PG, Cygankiewicz I, Corino VDA, Mainardi LT, Moss AJ, Zareba, W: Reduced short-term variability of RR intervals is associated with increased mortality in MADIT-II patients with atrial fibrillation. 2010, p. 719.

3. Corino VDA, Cygankiewicz I, Mainardi LT, Vazquez R, Bayes Genis A, Bayes de Luna A, Zareba W, Platonov PG: Reduced short-term variability of RR intervals during atrial fibrillation is associated with increased mortality in patients with congestive heart failure. Heart Rhythm Soc. Boston, 2012.

4. Platonov PG, Holmqvist F: Atrial fibrillatory rate and irregularity of ventricular response as predictors of clinical outcome in patients with atrial fibrillation. J Electrocardiol 2011;44:673-677.

5. Corino VDA, Cygankiewicz I, Mainardi LT, Stridh M, Zareba W, Vasquez R, Bayes de Luna A, Platonov PG: Association between atrial fibrillatory rate and heart rate variability in patients with atrial fibrillation and congestive heart failure. Ann Noninvasive Electrocardiol 2013;18:41-50.

6. Ulimoen SR, Enger S, Carlson J, Platonov PG, Pripp AH, Abdelnoor M, Arnesen H, Gjesdal K, Tveit A: Comparison of four singledrug regimens on ventricular rate and arrhythmia-related symptoms in patients with permanent atrial fibrillation. Am J Cardiol 2013;111: 225-230.

7. Task Force of the European Society of Cardiology and the North American Society of Pacing and Electrophysiology. 
Heart rate variability: Standards of measurement, physiological interpretation, and clinical use. Circulation 1996;93:10431065.

8. Pincus S: Approximate entropy (ApEn) as a complexity measure. Chaos Woodbury N 1995;5:110-117.

9. Richman JS, Moorman JR. Physiological time-series analysis using approximate entropy and sample entropy. Am J Physiol Heart Circ Physiol 2000;278:H2039-H2049.
10. Porta A, Baselli G, Liberati D, Montano N, Cogliati C, GnecchiRuscone T, Malliani A, Cerutti S: Measuring regularity by means of a corrected conditional entropy in sympathetic outflow. Biol Cybern. 1998;78:71-78.

11. Corino VDA, Holmqvist F, Mainardi LT, Platonov PG: Beta-blockade and A1-adenosine receptor agonist effects on atrial fibrillatory rate and atrioventricular conduction in patients with atrial fibrillation. Europace. 2014; 16:587-594. 